\title{
EL USO DEL DIBUJO PARA EL RECONOCIMIENTO Y LA EXPRESIÓN EMOCIONAL CONSTRUCTIVA DE ADOLESCENTES VICTIMAS DE CIBERACOSO
}

THE USE OF DRAWING FOR THE RECOGNITION AND CONSTRUCTIVE EMOTIONAL

\section{EXPRESSION OF TEENAGE VICTIMS OF CYBERBULLYING}

María del Consuelo Cervantes-Muñoz*, María Nieves González Valles*, Iris Rubí Monroy Velasco**

Universidad Autónoma de Ciudad Juárez*, Universidad Autónoma de Coahuila**, México.

Correspondencia: ccervantes67@hotmail.com

\section{RESUMEN}

El ciberacoso es un tipo de violencia entre iguales. Se presenta entre adolescentes y sus efectos pueden ir desde el sufrimiento psicológico al suicidio, entre otros. Ésta problemática revela que los estilos de afrontamiento poco adaptativos juegan un papel importante, pues en la adolescencia aún no se han desarrollado habilidades que permitan un apropiado manejo de las emociones en las relaciones interpersonales. De ahí la importancia de este estudio. Se implementa un programa psicoterapéutico grupal con cuatro adolescentes, para la promoción del reconocimiento, la comprensión y la expresión constructiva de emociones. En doce sesiones en las que se empleó el dibujo como técnica para facilitar la expresión verbal emocional, con el objetivo de sensibilizar a los adolescentes en su responsabilidad de sus respuestas. Los hallazgos obtenidos muestran que los participantes reportaron mejora sensible en 
el reconocimiento y la expresión constructiva de sus emociones en sus relaciones interpersonales familiares y escolares.

Palabras clave: adolescentes, psicoterapia, técnica del dibujo, reconocimiento emocional, expresión emocional.

\section{ABSTRACT}

Cyberbullying is a type of violence between equals. It occurs among adolescents and its effects can range from psychological suffering to suicide, among others. This problem reveals that non-adaptive coping styles play an important role, because most adolescents have not yet develop the set of skills that allows them to appropriately manage their emotions in interpersonal relationships, hence the importance of this study. A group psychotherapeutic program was implemented with four adolescents to promote recognition, understanding and constructive expression of emotions. In twelve sessions in which drawing was used as a technique to facilitate emotional verbal expression, with the aim of sensitizing adolescents in their responsibility for their responses. The results showed that the participants reported a noticeable improvement in the recognition and constructive expression of their emotions in their family and school interpersonal relationships.

Key words: adolescents, psychotherapy, drawing technique, emotional recognition, emotional expression.

\section{INTRODUCCIÓN}

Durante dos décadas se han realizado estudios orientados al problema del ciberacoso (Belsey, 2005; Finkelhor, Mitchel y Wolak, 2000). Muchas de las estrategias de prevención y tratamiento al acoso tradicional han sido 
también aplicadas al ciberacoso (Cowie, 2013). A pesar de los esfuerzos por disminuir el fenómeno de violencia que se presenta a través de las tecnologías de la información y la comunicación entre adolescentes, las cifras continúan creciendo (Mapua, 2018). Diversas investigaciones señalan que este problema ha sido predictor significativo de daño emocional, expresión de rabia, estrés, depresión, tristeza, confusión y en ocasiones ideas suicidas entre la población adolescente (Escobar-Echavarria, MontoyaGonzález, Restrepo-Bernal y Mejia-Rodríguez, 2017; Kowalski, Witte, Patchin e Hinduja, en Kowalski y Limber, 2013; Salmerón e Inostroza, 2017). Por lo que consideramos necesario un cambio en las estrategias de acercamiento a los efectos y de quienes lo experimentan con mayor frecuencia. Nuestro propósito estuvo orientado a incidir en las estructuras en dónde se experimenta y reproduce tal violencia.

Para entender la dinámica de la violencia y su reproducción, es necesario considerar los contextos en donde se genera y los componentes personales que se activan en la dinámica de las relaciones interpersonales. Álvarez (2009) plantea que, la violencia es una construcción producto de lo cultural derivada del aprendizaje, que se presenta como una discapacidad en la expresión de emociones y necesidades, y a nivel comportamental es nociva y trasgresora de los límites del otro. Como sucede en la dinámica del ciberacoso. El objetivo de este trabajo fue dotar a un grupo de adolescentes victimas de ciberacoso, de habilidades intrapersonales e interpersonales, para el reconocimiento y la expresión constructiva de sus emociones. Mediante un proceso psicoterapéutico grupal, en el que se empleó el dibujo como técnica facilitadora de la expresión emocional verbal y promotor de la expresión constructiva. 


\section{Consideraciones teóricas}

Sobre la adolescencia

"La palabra adolescente proviene del latín adultus (adulto) que en su extensión adulescens (haciéndose adulto) es la etapa entre la infancia y la adultez" (Almario, 2016:24). La Organización Mundial de la Salud (2018) la definen como etapa de crecimiento y desarrollo humano que se presenta entre los 10 y los 19 años, en la que hay un acelerado crecimiento físico y cambios psicológicos. Marsh (1989) plantea que, en esta etapa se genera una gran variedad de cambios que influyen en la vida física, cognitiva, emocional y social de la persona. Sorenson (en Hurlock, 1980) señala que es un período de transición constructivo necesario para el desarrollo del yo, en el que de acuerdo con Goossens (en Jackson y Goossens, 2016) transita de la dependencia infantil al estado adulto a través de las relaciones interpersonales. Se ha planteado que en el adolescente, el alcance y profundidad del darse cuenta de sí mismo es limitada debido a que está más interesado en lo externo y por consiguiente se encuentra un tanto disociado de sí mismo. Además, una de las principales metas en esta etapa es la madurez emocional (Almario, 2016) pues en el inicio incipiente de la identidad, son comunes la impulsividad y experimentación de cambios intempestivos de humor, debido a un escaso desarrollo de habilidades para el manejo emocional que se manifiesta como baja tolerancia a los conflictos internos y los refleja en el campo interpersonal (McConville, 1995). Cabe destacar que en esta etapa la capacidad de sentir y manifestar las emociones implica experimentar malestar aparejado con ansiedad y estrés. De manera que la posibilidad de vivir múltiples emociones que impactan de manera negativa al adolescente tanto en su vida personal como en lo social, se derivan de su escasa habilidad para conducir sus emociones de manera apropiada (Keltner, Moffit y Stouthamer-Loeber, 1995). Se ha visto que la falta de sensibilidad ante las propias emociones, obstaculiza 
el afrontamiento de situaciones conflictivas (Rieffe, Villanueva, Adrián y Górriz, 2009) como le sucede ante el ciberacoso.

\section{La mirada Gestalt Humanista}

Independientemente del modelo epistemológico desde el que se aborde la adolescencia, todos los teóricos coinciden en que la tarea principal de esta etapa es el desarrollo de la identidad del self, concepto de sí mismo o yo. Vista desde la Gestalt, la adolescencia es una etapa crucial para la evolución del contacto entre el self y el entorno (McConville, 1995). Además es importante que el adolescente aprenda a comprender las experiencias, los diferentes estados afectivos y la comunicación emocional para el apropiado desarrollo del self (Hauser y Safyer, 2010). Además se requiere entender el proceso de maduración en el que se reorganiza el campo de experiencia organismo-entorno, que implica compenetrarse y diferenciarse, para desarrollar la capacidad de mantener una frontera flexible que permita el intercambio del dar y tomar del entorno (Yontef, 2009). Así como distinguir entre el self y el no-self y organizar la relación entre ambos (McConville, 1995). Se ha planteado que el self se crea y recrea de manera constante y dinámica en la frontera de contracto organismo-entorno, que cambia con las necesidades dominantes del organismo y la presión de los estímulos del entorno. El self es el integrador y artífice de la vida, que juega un papel crucial para descubrir y construir los significados que motivan el crecimiento personal (Perls, Hefferline y Goodman, 2006; Yalom, 1986; Yontef, 2009). Se sabe que el adolescente hace contacto con el mundo no sólo a través de sus sentidos en la dinámica del presente (McLeod, 2010), sino también mediante su campo organismo-entorno en el área intrapsíquica e interpersonal. En la que se inicia la reorganización de las relaciones interpersonales y empieza a jugar un papel central la autoría de su self. Por su parte McConville (1995) plantea que, en la adolescencia temprana la frontera interpersonal se convierte en el foco de la experiencia, 
en donde se desarrolla primero la conciencia y articula un nuevo sentido de autodefinición. Se ha visto que en esta etapa existe un espacio en blanco cuando se le pide al adolescente que enfoque su atención en la frontera de contacto intrapsíquica, pues en esta fase recién ha salido de la infancia y aún está en lo que Kepner (1992) llama adaptación, pues se encuentra un tanto disociado de sí mismo y frecuentemente tiende a proyectar en el ambiente lo que experimenta internamente. Debido al escaso desarrollo de habilidades para el manejo emocional, el adolescente manifiesta baja tolerancia a los conflictos internos y suele reflejarlos en el campo interpersonal (McConville, 1995) como sucede en el ciberacoso. De ahí la importancia del trabajo expresivo de emociones en los adolescentes para la promoción de sus relaciones interpersonales no violentas.

\section{La psicoterapia grupal}

La psicoterapia grupal humanista es un método de tratamiento con múltiples bondades. Es un espacio para las relaciones interpersonales, que combinado con diversas técnicas busca que la persona se comprenda a sí misma a través del sentir, pensar y actuar de manera congruente (Weiner y Bordin en Weiner, 1992). Su propósito es promover la disminución de síntomas y/o problemas para generar crecimiento y desarrollo saludables (Perls, 2011). Así como ayudar a la auto-regulación de la persona a través de contacto con su entorno (Kriz, 2001), mejorando el autoconocimiento y la autoestima (Weiner y Bordin en Weiner, 1992). Esta modalidad de psicoterapia alienta a sus integrantes a compartir, retroalimentar y contrastar sus vivencias con las de otros para encontrar nuevas formas de ver y manejar sus problemas (Díaz, 2000). Además, facilita la identificación con la problemática de los demás y promueve la apertura, para que sus integrantes desarrollen sus habilidades y mejoren su capacidad para enfrentar sus dificultades (Delacroix, 2008). 
Este método de tratamiento tiene la cualidad de adaptarse a cualquier orientación teórica, ya que su eficacia radica en la posibilidad de establecer un sistema de retroalimentación y apoyo entre personas con problemas similares. Además, es un espacio en donde los participantes se despojan de su sensación de aislamiento y alienación, y mejoran sus habilidades sociales (Soutullo y Mardomingo, 2009). La dinámica que se genera en la psicoterapia grupal permite que las limitaciones de personalidad se superen mediante la capacidad de comunicación de la persona consigo misma y con los demás, en donde la experiencia y la expresión afectiva son juegan un papel esencial que favorece el trabajo con la consciencia, la promoción de la responsabilidad y el trabajo emocional en el aquí y ahora (Linn y Frauman en Linn y Garske, 1988). Más aún, la psicoterapia grupal pone énfasis en el potencial creativo de las personas y aprovecha el constante crecimiento en su dinámica de vida diaria, pues destaca la capacidad de identificar y satisfacer las propias necesidades (Cain en Cain y Seeman, 2000). Además, permite comprender la problemática personal y promueve la toma de consciencia, mediante el reconocimiento y el contacto con las emociones. Aunado a lo anterior, tenemos que su finalidad es ayudar al individuo a responsabilizarse y reestablecer su funcionamiento total e integrado, para modificar la conducta con sus sensaciones y sentimientos adyacentes (Polster y Polster, 2008). Finalmente, en la psicoterapia es esencial la incorporación de nuevas experiencias emocionales y el reforzamiento de vínculos entre memoria y emoción, acompañado de la práctica de nuevas formas de comportamiento en nuevos contextos (Lane, Ryan, Nadel, y Greenberg, 2015; Latner, 2007), como las relaciones interpersonales mediante el uso de las nuevas tecnologías. 


\section{Sobre el dibujo}

Se ha encontrado que un papel en blanco es un espacio ilimitado para la expresión de la persona que puede ayudarle a satisfacer necesidades de comunicación (Stern, 1977). Es un medio por el cual se expresa lo que se siente, de manera que influye en la autoestima y el autoconcepto a la vez que los revela (López, 2015). El dibujo se emplea para la expresión de cualquier tema que resulta difícil o doloroso de expresar verbalmente (Cornejo, 2014; Oaklander, 2006), que no se ha podido expresar de otra manera (Stern, 1977). Se sabe que el dibujo promueve un estado emocionalmente sano (Read, 1969), pues dibujar los sentimientos puede generar alivio para la persona en conflicto y es una forma de expresar emociones, pensamientos, intereses, deseos e impulsos que acontece en el interior y en el entorno del adolescente. (Oaklander, 2010). Por lo anterior y debido a la dificultad de los adolescentes para el establecimiento de contacto emocional interno, se ha visto que el dibujo es un facilitador de dicho contacto y que además promueve la expresión verbal y emocional (Cervantes-Muñoz, 2019) y "supone una de las mejores medicinas para el dolor psíquico" (Hernández, 2006, p. 83).

\section{Sobre las emociones}

Las emociones determinan la calidad de nuestra vida (Ekman, 2003) y son centrales en las formas comunicativas de las personas a nivel interpersonal. En la actualidad, existen múltiples teorías sobre las emociones, (Cannon, 1929; Darwin, 1984; James, 1994 y; Plutchik, 1991) $\mathrm{y}$ diversos investigadores coinciden en que hay una estrecha interacción entre emoción y cognición (Damasio, 2010; Greenberg y Paivio, 1997). Se ha visto que, las emociones influyen en la actividad cognitiva y esta a su vez puede ser una fuente de emociones (Damasio, 2005). Para James (1994) la manifestación de las emociones es la combinación de sensaciones, 
creencias y reacciones con funciones adaptativas. Palmero (2000) señala que las emociones que cada persona experimenta en un determinado evento están determinadas por la valoración que hace del mismo. AL respecto McConville (1995) plantea que las emociones están ligadas a las necesidades más esenciales del ser humano y forman parte de sus procesos cognitivos. Para Sroufe (2000) resultan fundamentales debido a que, son el corazón de las relaciones cercanas y juegan un papel preponderante en la negociación y el manejo del conflicto. Además, se ha visto que para que el adolescente encause de manera adecuada sus emociones, es importante que aprenda a reconocer las necesidades que les subyacen y con base en su toma de consciencia y responsabilidad las exprese de manera constructiva.

\section{MÉTODO}

Los participantes del grupo fueron cuatro estudiantes de primer grado de secundaria, dos hombres y dos mujeres, de nivel socioeconómico bajo, ambos padres trabajan, por lo que los adolescentes están al cuidado del personal de una estancia infantil. En dónde permanecen de lunes a viernes después del horario escolar. Generalmente sus padres van por ellos entre las 18:00 y las 19:00 horas después de su jornada laboral.

Tabla 1. Características de los integrantes del grupo

\begin{tabular}{|cccc|}
\hline Seudónimo & Sexo & Edad & Grado escolar \\
\hline VAN & M & 11 & $1^{\circ}$ de secundaria \\
\hline MAR & H & 11 & $1^{\circ}$ de secundaria \\
\hline EST & M & 12 & $1^{\circ}$ de secundaria \\
\hline PAS & H & 12 & $1^{\circ}$ de secundaria \\
\hline & Cervantes-Muñoz, MC (2019). & \\
\hline
\end{tabular}


En la estancia los adolescentes reciben alimentos en la hora de la comida y de la merienda. El personal los asesora en la realización de tareas escolares y al finalizarlas realizan actividades lúdicas o ven algún video. Los adolescentes llevan más de cinco años asistiendo a este lugar debido a que sus padres no los pueden cuidar por sus horarios laborales. Criterios de inclusión: que fueran adolescentes víctimas de ciberacoso, que aceptaran participar en el estudio y que sus padres firmaran el consentimiento informado. En la Tabla 1. Se muestran las características de los integrantes del grupo y se emplean seudónimos con la finalidad de guardar la confidencialidad de sus nombres y expresiones vertidas en este trabajo, como lo indica el Art. 68 del Código Ético del Psicólogo (SMP, 2009).

Obtención de datos: debido a que al momento de realizar la investigación las investigadoras no contaban con herramientas desarrolladas y/o validadas en población mexicana respecto del tema a investigar, determinaron por consenso emplear el Cuestionario de Acoso a través de los Medios Electrónicos elaborado por Ortega, Calmaestra y MoraMerchán (2007). Lo hicieron bajo la consideración del contenido de los elementos precisos para la exploración del problema y que resulta adecuado a la población, además de brindarnos la posibilidad de obtener datos de la problemática de manera precisa. Este instrumento es de tipo mixto, requiere de respuestas cerradas y otras abiertas. Está estructurado en tres secciones, la primera indica el acceso de la persona a las nuevas tecnologías y si ha sido ciberacosado. La segunda sección, recaba datos sociodemográficos y la tercera sección está integrada por 7 preguntas con respuestas cerradas de selección múltiple y una pregunta abierta. La pregunta 1 se asocia a las emociones derivadas del ciberacoso. La 2 señala las respuestas ante el ciberacoso. La 3 se refiere a la frecuencia con la que la persona ha sido acosada a través de los medios electrónicos. La 4 
contiene las explicaciones construidas ante el ciberacoso. La 5 muestra las formas de afrontamiento ante el ciberacoso. La 6 revela las soluciones que la persona ha implementado ante el ciberacoso. La pregunta 7 indica si la persona que vive ciberacoso conoce de algún(os) amigo(s) que haya(n) sido ciberacosado(s). Es un instrumento auto-administrado en el que las respuestas son marcadas por las personas participantes (Hernández, et al, 2016). Se aplicó una entrevista inicial semiestructurada para conocer la manera en que los adolescentes reaccionan ante las emociones que les genera su entorno familiar y escolar. Se implementó un cuestionario final para conocer los cambios identificados por los adolescentes en cuanto a su reacción emocional en su entorno familiar y escolar.

Además, se realizaron audio-grabaciones para recopilar las respuestas verbales de los participantes en el proceso, así como notas de campo para registrar aspectos fenomenológicos de las respuestas de los adolescentes en cada una de las sesiones.

Este trabajo se aborda desde la perspectiva cualitativa, su propósito es la comprensión del proceso de vinculación entre personas y situaciones en determinadas circunstancias, así como los patrones que se desarrollan en un tiempo y espacio determinado (Hernández, Fernández y Baptista, 2016). Los métodos cualitativos al ser humanistas (Taylor y Bogdan, 1990) plantean conocer a las personas a partir de la cotidianeidad en la que viven y buscan aprender conceptos como: sufrimiento, frustración, amor, entre otros y mantenerse próximos al mundo empírico, pues toma en cuenta las experiencias, comportamientos, emociones y sentimientos de los participantes (Denzin y Lincoln, 2018; Strauss y Colbin, 2002). Su alcance es descriptivo con diseño pre-experimental, exploratorio. 
La labor psicoterapéutica fue realizada por la autora principal de este trabajo, quien es psicóloga con más de quince años de experiencia en el área de psicología clínica y psicoterapia, cuenta con conocimiento especializado en la evaluación de las emociones expresadas en estudios de investigación.

Intervención: en la Tabla 1 se muestran los temas abordados durante el proceso psicoterapéutico y el propósito de cada uno de ellos.

Tabla 1. Temas abordados durante el proceso psicoterapéutico

\begin{tabular}{ll}
\hline \multicolumn{1}{c}{ Tema } & \multicolumn{1}{c}{ Propósito } \\
\hline Identidad del adolescentes & Identificar sus preferencias, lo que disfrutan hacer en su vida cotidiana lo que los describe y define como \\
& adolescentes. \\
\hline Conocimiento de emociones & Explorar el reconocimiento de sus emociones, cómo las usan y como les ayudan en sus relaciones \\
\hline interpersonales cotidianas.
\end{tabular}

Cervantes-Muñoz, MC (2019).

\section{ANÁLISIS DE DATOS}

Los datos aquí presentados se extraen de la tesis de grado de la autora de este trabajo, cuyo título es: "Efectos de la Psicoterapia Grupal Humanista en el reconocimiento y expresión emocional constructiva de adolescentes 
víctimas de ciberacoso". Sin embargo lo que aquí se presenta destaca el empleo de del dibujo como técnica facilitadora del reconocimiento en un contexto de psicoterapia grupal.

Cuando se realiza el análisis de datos derivados de un estudio cualitativo, es imprescindible emplear métodos rigurosos en su tratamiento, con la finalidad de hacer confiables los hallazgos de la investigación (May y Pope, 1995). Por lo que en éste trabajo se empleó el método de análisis clásico de contenido de la información derivada de la aplicación de los instrumentos empleados para la recolección de los datos (entrevista, cuestionarios y grabaciones). Se eligió este método debido a que contempla el análisis del contenido manifiesto -lo que el participante declara-, así como el contenido latente -lo que surge de la interpretación del que investiga- (Berelson, 1992). Diversos autores han planteado que el análisis clásico de contenido forma parte de los análisis textuales y puede efectuarse mediante categorías deductivas y categorías inductivas (Díaz y Navarro, 1998; Hsieh y Shannon, 2005). Para este trabajo se emplearon las categorías inductivas debido a que aluden a las experiencias peculiares de los informantes así como de los conceptos individuales surgidos de lo observado (Mayring, 2000), como es el caso del conjunto de respuestas verbales y escritas vertidas por los adolescentes durante la entrevista, el Cuestionario de Acoso, las expresiones en su participación durante el proceso psicoterapéutico y lo observado por las autoras de este trabajo.

El procedimiento para la elaboración de las categorías, una sola investigadora se encargó de la lectura de los datos de manera reiterada, con el propósito de tener un claro entendimiento del conjunto de datos. Con base en ello, planteó un esquema inicial de codificación de forma inductiva. Este esquema fue discutido con dos investigadoras mas, es decir se llevó a cabo un proceso de triangulación y la codificación de los datos 
fue contrastada por las tres investigadoras. Posteriormente los códigos se asignaron a las diferentes categorías tomando como base su relación y el grado de vinculación. De tal manera que, las categorías emergentes fueron reunidas en grupos significativos y de esta forma quedaron definida las cuatro categorías que se muestran en los resultados.

Para el análisis e interpretación de los datos derivados de cada una de las sesiones se atendió a dos temas principales planteados al inicio de este estudio el Reconocimiento Emocional y la Expresión Emocional Constructiva, así como las emociones de enojo y tristeza debido a que fueron emociones predominantes en el diagnóstico.

En términos generales tenemos que el cuestionario permitió explorar las respuestas que los adolescentes presentaron ante situaciones de ciberacoso, es decir, dio cuenta de las experiencias y respuestas emocionales, verbales y comportamentales. La entrevista permitió explorar de manera detallada las experiencias emocionales y respuestas comportamentales derivadas del ciberacoso. El dibujo permitió ilustrar y a la vez facilitar la verbalización de las emociones experimentadas durante el proceso terapéutico enfocado en las emociones en general y en el reconocimiento y la expresión constructiva en particular. La información recabada durante el proceso fue audio-grabado y posteriormente transcrita. Toda la información ya capturada fue procesada mediante un programa construido ex profeso para el análisis de datos que permitió conocer la frecuencia con la que se presentaron determinadas palabras, en este caso asociadas a la expresión emocional como: enojo, enojado, enfado, enfadado, furioso, tristeza, 
depresión, desanimado, aislado, encerrado, etc. palabras que denotan estados de ánimo asociados a emociones específicas. También se obtuvo un reporte de palabras apareadas. Cabe mencionar que además se abordaron emociones como la alegría y el miedo pero que debido a la naturaleza de la investigación el trabajo se centró principalmente a la facilitación del reconocimiento de emociones que se experimentaron como desagradables y su expresión constructiva. En la tabla 2 se muestra el eje temático en función de cuatro categorías y la intervención.

En lo que respecta al objetivo del uso del dibujo, se empleó como instrumento que facilitó la obtención de información gráfica verbal respecto de las emociones experimentadas no sólo durante el ciberacoso y durante situaciones similares en la vida diaria en la que han sido objeto de abuso por parte de otros compañeros. Se observó que esta técnica expresiva permitió a los adolescentes expresar de forma gráfica acompañada de la expresión verbal el cómo este grupo de adolescentes experimentaron sus emociones en general y la manera en que respondieron ante dichas experiencias. 
Tabla 2. Eje y categorías de Análisis

\begin{tabular}{|c|c|}
\hline Eje & Tategorías \\
\hline $\begin{array}{l}\frac{\text { El ciberacoso es la }}{\text { violencia entre iguales }} \\
\text { mediante insultos, } \\
\text { vejaciones, humillaciones, } \\
\text { amenazas, chantaje, } \\
\text { etcétera, utilizando un } \\
\text { canal tecnológico }\end{array}$ & $\begin{array}{l}\text { * Reconocimiento Emocional } \underline{\text { RE }} \text { Capacidad de la } \\
\text { persona de identificar lo que siente en sí misma } \\
\text { y percibir las emociones expresadas por otras } \\
\text { personas. } \\
\text { *Expresión Emocional Constructiva EEC estilo } \\
\text { comunicativo de dar a conocer a los demás las } \\
\text { necesidades y sentimientos mediante diversas } \\
\text { formas de conducta verbal o comportamental que } \\
\text { posibilitan crear relaciones interpersonales sanas } \\
\text { basadas en el respeto. } \\
\text { *El enojo es una alteración anímica donde se } \\
\text { experimenta irritación, rabia y afán de revancha } \\
\text { o violencia acompanada de una sensación de } \\
\text { invasión y amenaza a la integridad y a la propia } \\
\text { existencia, que puede estar aparejada con } \\
\text { sensación de fuerza y poder. } \\
\text { *La tristeza es un sentimiento de dolor anímico } \\
\text { producido por un suceso desfavorable, se } \\
\text { manifiesta como un estado de ánimo pesimista, de } \\
\text { insatisfacción y con tendencia al llanto. }\end{array}$ \\
\hline
\end{tabular}

*Cervantes-Muñoz, MC (2019).
Intervención

El dibujo se empleó como un elemento facilitador de la expresión de la experiencia emocional de los adolescentes y el reconocimiento de sus respuestas motivadas por sus emociones en relación con su entorno. Se trabajó mediante la reflexión y el darse cuenta del cómo expresan lo que sienten, en su experiencia. De manera que se observaron cambios orientados a la expresión no violenta de sus emociones, el fortalecimiento de su autoestima, la exploración de su enojo y tristeza, así como la empatía y su experiencia a lo lardo de la línea del proceso.

\section{RESULTADOS}

De las respuestas que los adolescentes vertieron en el Cuestionario de Acoso a Través de los Medios Electrónicos, se encontró que las emociones de tristeza y enojo predominaron. Las respuestas comportamentales derivadas de la exposición al ciberacoso fueron MAR:H:11 "ignorar lo que estaba pasando", VAN:M:11 "se lo he dicho a mis padres, se lo he dicho a mis amigos". En las explicaciones construidas reportaron que habían sido ciberacosado Mar y EST "porque querían molestarme” y VAN:M:11 Y MAR:H:11 “por hacerme una broma”. En las conductas de afrontamiento la mayoría respondió VAN:M:11 "reduje su número de amigos en las redes sociales". En cuanto a la información recabada 
durante las entrevistas se encontró que los adolescentes suelen tener respuesta exaltadas y desproporcionadas ante eventos que les ocasionan enojo. La mayoría de las veces suelen responder de manera violenta, que por lo general es en modalidad de violencia física y/o verbal, con una clara tendencia a incrementar sus problemas y conflictos con estas respuestas. Además reportaron que en sus familias es común esa forma de respuesta ante el conflicto. Esto último coincide con lo planteado por Álvarez (2009) acerca de que la violencia es una construcción producto de lo cultural que deriva del aprendizaje y se presenta como una discapacidad en la expresión de emociones o necesidades.

A continuación se muestran algunos de los dibujos que los adolescentes realizaron durante las sesiones del proceso psicoterapéutico que dan cuenta de los diferentes temas abordados. Son en principio la expresión gráfica de cómo se perciben a sí mismos y sus experiencias emocionales. Durante las sesiones psicoterapéuticas la expresión a través del dibujo promovió en los adolescentes la puesta en práctica de centrar su atención en la experiencia de sí mismos y facilitó que comunicaran y dieran a conocer cómo se experimentan y la forma en que expresan eso están sintiendo internamente.

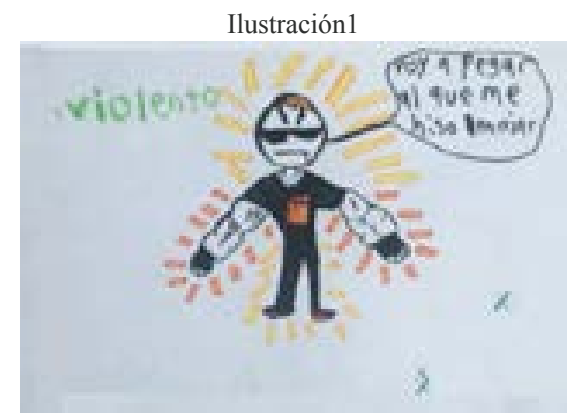

Cervantes-Muñoz, MC (2019). Ilustración 1. MAR así me siento cuando me enojo. 
Los adolescentes hicieron referencia al significado personal estrechamente asociado a los elementos gráficos, a continuación se presentan una serie de expresiones verbales que acompañaron la reflexión y verbalización en torno a sus dibujos: del enojo MAR adolescente hombre de 11 años (MAR:H:11) Ilustración 1:

"Cuando me enojo, me enojo de verdad y me pongo furioso y aviento todo para todos lados y comienzo a golpear lo que encuentro" "cuando me enojo, lo siento en todo mi cuerpo, pero es más en mis brazos y manos. Golpeo al que me hace enojar". "Aqui escribo violento porque me pongo muy violento cuando alguien me insulta. Estas rayas rojas en los brazos son porque me dan ganas de aventar todo lo que encuentro y golpear. También las rayas amarillar significan que siento enojo en mi cabeza y en mis piernas, pero no es tan fuerte. Mi coraje siempre sale por mis brazos, por eso los dibujé muy musculosos porque ahi está toda mi fuerza", "Me gustaría que cuando me enojo todo fuera más controlable”.

Ilustración 2

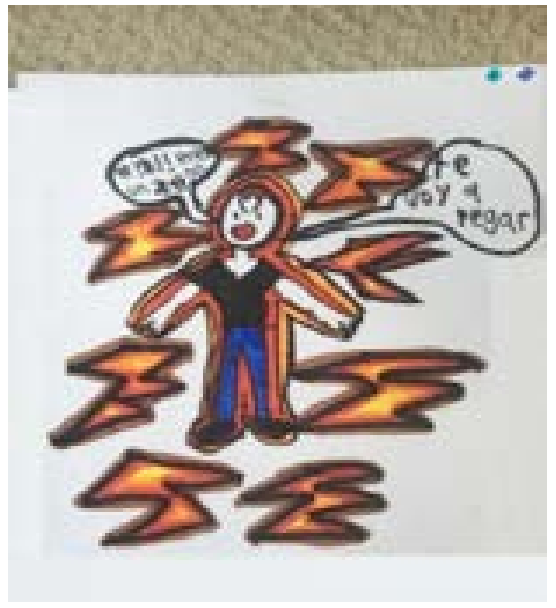

Cervantes-Muñoz, MC (2019). Ilustración 8. PAS así me siento cuando me enojo. 
PAS adolescente hombre de 12 años (PAS:H:12) Ilustración 2:

"Cuando me enojo siento mucho calor en todo mi cuerpo, es como una energía que sale por todos lados, aunque si siento ganas de golpear, golpeo un balón y siento que descargo todo mi enojo, mi coraje y (después) me calmo, en mis pies es en donde primero sale. También a través de mis manos, cierro mis puños y golpeo en la cama y ahí saco todo mi coraje".

Ilustración 3

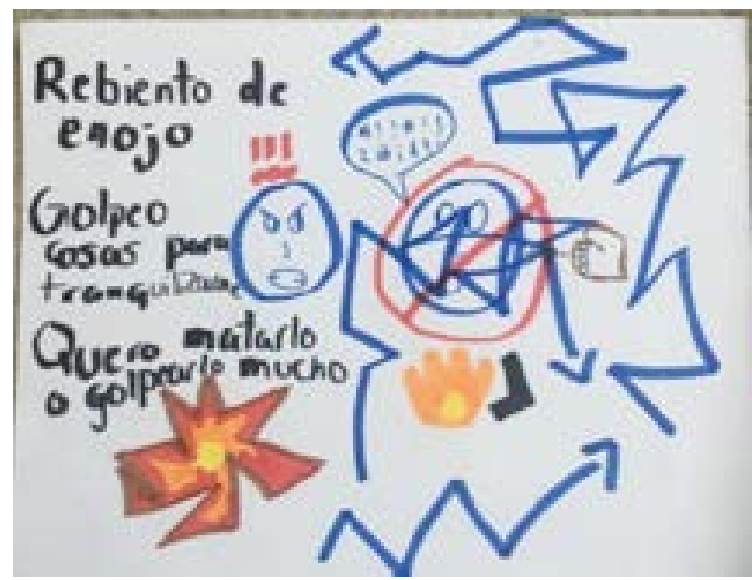

Cervantes-Muñoz, MC (2019). Ilustración 10. Expresión de enojo de VAN

\section{VAN adolescente mujer de 11 años (VAN:M:11) Ilustración 3:}

"Cuando alguien me insulta yo siento que reviento de enojo, a veces golpeo cosas para tranquilizarme Este dibujo amarillo, naranja y rojo es una fuerza que siento cuando alguien me hace enojar, ... a veces siento muchas ganas de decir groserías a la persona que me insulta. Esta flama es el enojo, es algo que arde dentro de mí y aqui dibujé una pistola, porque algunas veces cuando alguien me ha dañado y siento mucho enojo, me gustaría tener una pistola y como lo dije me dan ganas de matarlo, yo sé que eso no está bien porque me descontrolo toda y que estaría 
muy mal que yo dañara a alguien con un arma, pero es muy grande mi enojo. Me gustaría que cuando me enojo todo fuera más controlable”.

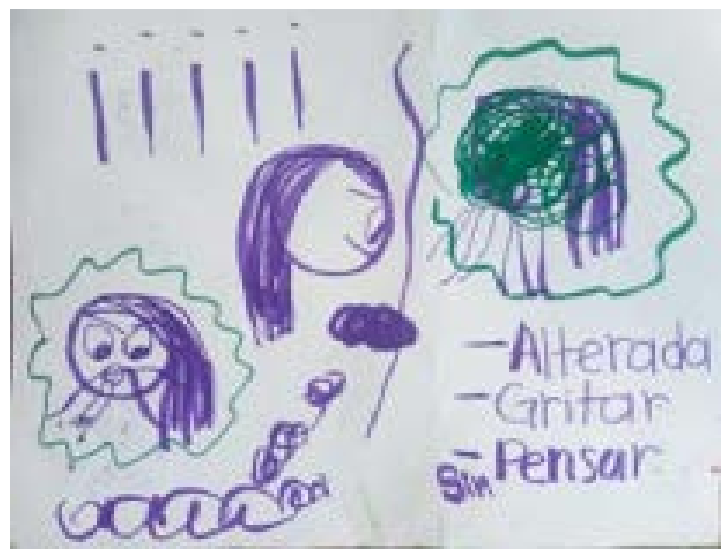

Cervantes-Muñoz, MC (2019). Ilustración 11. Expresión de enojo de EST

EST adolescente mujer de 12 años (EST:M:12) Ilustración 4: “

Cuando me enojo me siento alterada y casi siempre comienzo a gritar y digo cosas que me ayudan a sacar mi enojo”. "...aparte de poner mi cara de enojada me pongo a gritar, esto verde es como todo el coraje que siento y por eso grito". "dibujo nubes de humo porque cuando me enojo siento que echo humo, como si me estuviera quemando y mi enojo sale gritando". Como lo muestran las expresiones de los adolescentes múltiples emociones, en este caso la experiencia de enojo impacta de manera negativa al adolescente tanto en su vida personal como social, debido a la inhabilidad para conducirla de manera apropiada (Keltner, Moffit y Stouthamer-Loeber, 1995), y que como plantea McConville (1995) debido al escaso desarrollo de habilidades para el manejo emocional, el adolescente manifiesta baja tolerancia y lo refleja en su campo interpersonal. En las expresiones vertidas por los adolescentes como respuesta a su experiencia 
de enojo notamos lo señalado por Álvarez (2009) en el sentido de que la violencia en su nivel comportamental se presenta como nociva y por acción u omisión trasgrede los límites del otro.

Si atendemos a los elementos gráficos en relación con las expresiones verbales encontramos que en el proceso de expresión los adolescentes añaden elementos que no necesariamente se encuentran expresados en el dibujo, Como sus sensaciones corporales y sus respuestas comportamentales como: "golpear", "aventar todo", "asilarme", etc. y estos elementos muestran que lo grafico facilita expresiones emocionales verbales que hacen alusión a la conexión de la emoción con la parte comportamental. Esto coincide con lo planteado por Oaklander (2010) en el sentido de que el dibujo es una forma de expresar emociones, pensamientos, intereses, deseos e impulsos que acontece en el interior $\mathrm{y}$ en el entorno del adolescente.

De igual manera lo hicieron en su experiencia de tristeza, alegría y miedo, que por cuestión de espacio se omiten en este artículo.

Para contrastar las respuestas del antes y el después del proceso psicoterapéutico se muestran a continuación algunas de las expresiones de los adolescentes al final del proceso: (VAN:M:11) “...no es malo enojarse, lo malo es no saber manejar el enojo (para) resolver los problemas..." (MAR:H11) "hay que buscar otras formas menos violentas de expresar el enojo". (VAN:M:11) "que es bueno hablar de lo que nos sucede cuando nos enojamos porque es una forma de que nos puedan entender las personas con las que vivimos", (MAR:H11) "hay que buscar otras formas menos violentas de expresar el enojo", (PAS:H:12) "necesito buscar actividades cuando me enojo para bajarlo y después hablar", (EST:M:12) "que mejor me encierro en mi cuarto mientras se me baja el enojo y cuando ya esté 
calmada regreso a hablar con la persona que me hizo enojar, en lugar de gritar de cosas". Y en relación al ciberacoso (MAR:H11) "pues muy fácil, bloquearlo y borrarlos", (PAS:H:12) "no hacerles caso, si es de un desconocido ni leerlo, borrarlo y bloquearlo, y si es conocido ir a la dirección de la escuela y hablar con los maestros y la directora para que lo castiguen", (VAN:M:11) "borrar todos los mensajes y bloquear los números, aunque sean de personas conocidas", (EST:M:12) "sólo tener en nuestras redes sociales a personas que conocemos y que son nuestros amigos, para que no tengamos problemas".

Tabla 3. Resultado de la entrevista inicial

\begin{tabular}{clccccccc} 
Part. & \multicolumn{2}{c}{ RE } & \multicolumn{2}{c}{ EEC } & \multicolumn{2}{c}{ Expresión enojo } & \multicolumn{2}{c}{ Expresión tristeza } \\
& Propio & Otros & \multicolumn{2}{c}{ Verbal Conducta } & \multicolumn{2}{c}{ Verbal Conducta } & \multicolumn{2}{c}{ Verbal Conducta } \\
\hline VAN & $\mathrm{Si}$ & $\mathrm{No}$ & $\mathrm{No}$ & $\mathrm{No}$ & $\mathrm{Si}$ & $\mathrm{Si}$ & $\mathrm{No}$ & $\mathrm{Si}$ \\
\hline MAR & $\mathrm{Si}$ & $\mathrm{No}$ & $\mathrm{No}$ & $\mathrm{No}$ & $\mathrm{Si}$ & $\mathrm{Si}$ & $\mathrm{No}$ & $\mathrm{Si}$ \\
\hline EST & $\mathrm{Si}$ & $\mathrm{No}$ & $\mathrm{No}$ & $\mathrm{No}$ & $\mathrm{Si}$ & $\mathrm{Si}$ & $\mathrm{No}$ & $\mathrm{Si}$ \\
\hline PAS & $\mathrm{Si}$ & $\mathrm{No}$ & $\mathrm{No}$ & $\mathrm{No}$ & $\mathrm{Si}$ & $\mathrm{Si}$ & $\mathrm{No}$ & $\mathrm{Si}$ \\
\hline
\end{tabular}

La Tabla 3 muestra los datos de la entrevista inicial a los participantes. Se puede apreciar que todos revelaron que son capaces de reconocer las emociones que ellos experimentan. Sin embargo, en el RE reportaron que les cuesta trabajo identificar las emociones de otras personas. En lo referente a EEC no hubo respuestas asociadas a esta categoría. Ante la experiencia de enojo suelen expresarse con groserías, gritos y golpes. En la expresión de su tristeza, reportaron que se quedaban callados, negaban que algo les sucedía y se aislaban.

Tabla 4. Reconocimiento Emocional

$\begin{array}{cc}\text { Participante } & \text { RE } \\ \text { VAN } & \text { Abundante }\end{array}$




\begin{tabular}{|cc} 
MAR & Escaso \\
\hline EST & Escaso \\
\hline PAS & Abundante \\
\hline
\end{tabular}

En la Tabla 4. Se muestran los datos derivados del trabajo con el Reconocimiento Emocional y encontramos que tanto VAN:M:11 como PAS:H:12 mostraron un abundante reconocimiento de emociones en sus compañeros. En contraste con MAR:H:11 y EST:M:12 en quienes el RE fue escaso. Sin embargo, reportaron que esta sesión les permitió darse cuenta de la importancia de poner atención a las expresiones emocionales de los demás para entablar una comunicación más efectiva y completa. Y con base en el trabajo terapéutico se logró superar la limitación planteada por McConvill (1995) es en cuanto a que en el adolescente, el alcance y profundidad del darse cuenta de sí mismo es limitada debido a que está más interesado en lo externo y por consiguiente se encuentra un tanto disociado de sí mismo. El trabajo por tanto se centró en que los adolescentes pusieran su atención en las experiencias emocionales de sí mismo con el propósito de identificar sus estados emocionales y los de sus compañeros para facilitar su comunicación más efectiva. Sobre todo, que les permita establecer relaciones más armoniosas con las personas que les rodean.

Tabla 5. Reconocimiento y Expresión Emocional

\begin{tabular}{ccccc} 
Part. & \multicolumn{2}{c}{ RE } & \multicolumn{2}{c}{ EEC } \\
& \multicolumn{2}{c}{ Propio Otros } & \multicolumn{2}{c}{ Verbal Conducta } \\
VAN & $\mathrm{Si}$ & $\mathrm{Si}$ & $\mathrm{Si}$ & $\mathrm{Si}$ \\
MAR & $\mathrm{Si}$ & $\mathrm{Si}$ & $\mathrm{Si}$ & $\mathrm{Si}$ \\
EST & $\mathrm{Si}$ & $\mathrm{Si}$ & $\mathrm{Si}$ & $\mathrm{Si}$ \\
PAS & $\mathrm{Si}$ & $\mathrm{Si}$ & $\mathrm{Si}$ & $\mathrm{Si}$ \\
\hline
\end{tabular}

En la tabla 5. Hace referencia a las respuestas de los adolescentes cuando realizan el reconocimiento y expresión emocional respecto de sí mismos y 
en relación con su autoestima. Podemos observar que al trabajar este tema los cuatro integrantes del grupo fueron capaces de reconocer emociones propias y las de otros compañeros. Aquí podemos ver lo planteado por diversos autores en relación a que la falta de sensibilidad ante las propias emociones, puede ser un factor que obstaculice el afrontamiento de situaciones conflictivas (Rieffe, Villanueva, Adrián y Górriz, 2009). En cuanto a EEC todos mostraron que son capaces de expresarse de manera favorable de sí mismos, así como de procurar su autocuidado.

Tabla 6. Reconocimiento y expresión del enojo

\begin{tabular}{clccccc} 
Part. & \multicolumn{2}{c}{ RE } & \multicolumn{2}{c}{ EEC } & \multicolumn{2}{c}{ Expresión enojo } \\
& \multicolumn{2}{c}{ Propio } & Otros & \multicolumn{2}{c}{ Verbal Conducta } & \multicolumn{2}{c}{ Verbal Conducta } \\
\hline VAN & $\mathrm{Si}$ & $\mathrm{Si}$ & $\mathrm{No}$ & $\mathrm{No}$ & $\mathrm{Si}$ & $\mathrm{Si}$ \\
\hline MAR & $\mathrm{Si}$ & $\mathrm{No}$ & $\mathrm{No}$ & $\mathrm{No}$ & $\mathrm{Si}$ & $\mathrm{Si}$ \\
\hline EST & $\mathrm{Si}$ & $\mathrm{Si}$ & $\mathrm{No}$ & $\mathrm{No}$ & $\mathrm{Si}$ & $\mathrm{Si}$ \\
PAS & $\mathrm{Si}$ & $\mathrm{Si}$ & $\mathrm{No}$ & $\mathrm{No}$ & $\mathrm{Si}$ & $\mathrm{Si}$ \\
\hline
\end{tabular}

La tabla 6. Muestra que derivado del reconocimiento y expresión emocional del enojo. Se observa que VAN:M:11, EST:M:12 y PAS:H:12 fueron capaces de reconocer el enojo en sí mismos y en otros, mientras que MAR:H:11 sólo fue capaz de reconocer el enojo en sí mismo y no reconoció el enojo de los demás. En la EEC del enojo se pudo observar que todos fueron capaces de expresar su enojo de manera verbal y conductual. Se hizo evidente en sus dibujos que cuando se enojan, sus expresiones físicas y verbales suelen ser violentas. Esto coincide con lo planteado por Muñoz- 
Polit (2010) en el sentido de que cuando la persona experimenta enojo está percibiendo una invasión a sus propios límites y necesita defenderse y que dentro de las estrategias para la defensa está el ataque.

Tabla 7. Reconocimiento y expresión de la tristeza

\begin{tabular}{|c|c|c|c|c|c|}
\hline \multirow{3}{*}{$\begin{array}{l}\text { Part. } \\
\text { VAN }\end{array}$} & \multirow{3}{*}{$\begin{array}{c}\text { RE } \\
\text { Propio } \\
\text { Si }\end{array}$} & \multicolumn{2}{|c|}{ EEC } & \multicolumn{2}{|c|}{ Expresión tristeza } \\
\hline & & \multicolumn{2}{|c|}{ Verbal Conducta } & & Conducta \\
\hline & & No & No & $\mathrm{Si}$ & $\mathrm{Si}$ \\
\hline MAR & $\mathrm{Si}$ & No & No & $\mathrm{Si}$ & $\mathrm{Si}$ \\
\hline EST & $\mathrm{Si}$ & No & No & $\mathrm{Si}$ & $\mathrm{Si}$ \\
\hline PAS & $\mathrm{Si}$ & No & No & $\mathrm{Si}$ & $\mathrm{Si}$ \\
\hline
\end{tabular}

En la Tabla 7. Podemos ver que los resultados nos muestran que al trabajar en el reconocimiento y la expresión de la tristeza en este grupo de adolescentes todos son capaces de reconocer cuando experimentan tristeza y describir claramente las situaciones que los han entristecido así como identificar sus conductas. En esta fase del trabajo se pudo observar lo planteado por diversos autores en el sentido de que el dibujo facilita que el adolescente hable de algún tema que le resulta difícil de expresar verbalmente (Cornejo, 2014; Oaklander, 2006). Sin embargo, reportaron mediante sus expresiones verbales y conductuales que no se han podido expresar de forma constructiva porque para ellos es una emoción difícil de manejar. Así lo manifiesta VAN:M:11 cuando expresa "de mis debilidades está la tristeza, porque es algo con lo que me siento mal y eso no me gusta”. ES:M:12 “...también me encierro en mi cuarto para que no me vean llorar". En estas expresiones se puede ver claramente que la tristeza es un sentimiento de dolor anímico producido por un suceso desfavorable que se manifiesta como un estado de ánimo pesimista, de insatisfacción y con tendencia al llanto como se define esta categoría en la Tabla 2.

Tabla 8. Reconocimiento de las propias emociones

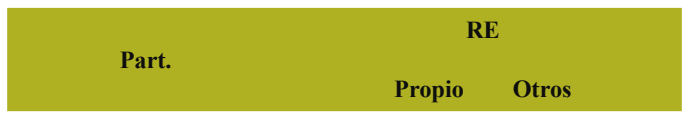




\begin{tabular}{|cll|}
\hline VAN & $\mathrm{Si}$ & $\mathrm{Si}$ \\
\hline MAR & $\mathrm{Si}$ & $\mathrm{Si}$ \\
\hline EST & $\mathrm{Si}$ & $\mathrm{Si}$ \\
\hline PAS & $\mathrm{Si}$ & $\mathrm{Si}$ \\
\hline
\end{tabular}

En cuanto al trabajo con la empatía y las propias emociones, los datos de la Tabla 8 nos muestran que, una vez que los adolescentes pusieron en práctica su atención en las propias emociones y en las de los demás con la ayuda del dibujo pudieron reconocer sus propias emociones y las de sus compañeros. Reportaron que la atención focalizada en las emociones de sus compañeros les ayudó a ser empáticos entre ellos.

Tabla 9. Resultados globales del Reconocimiento y Expresión Emocional

\begin{tabular}{clccccccc} 
Part. & \multicolumn{2}{c}{ RE } & \multicolumn{2}{c}{ EEC } & \multicolumn{2}{c}{ Expresión enojo } & \multicolumn{2}{c}{ Expresión tristeza } \\
& Propio Otros & \multicolumn{2}{c}{ Verbal Conducta } & \multicolumn{2}{c}{ Verbal Conducta } & \multicolumn{2}{c}{ Verbal Conducta } \\
\hline VAN & $\mathrm{Si}$ & $\mathrm{Si}$ & $\mathrm{Si}$ & $\mathrm{Si}$ & $\mathrm{Si}$ & $\mathrm{Si}$ & $\mathrm{Si}$ & $\mathrm{Si}$ \\
MAR & $\mathrm{Si}$ & $\mathrm{Si}$ & $\mathrm{Si}$ & $\mathrm{Si}$ & $\mathrm{Si}$ & $\mathrm{Si}$ & $\mathrm{Si}$ & $\mathrm{Si}$ \\
EST & $\mathrm{Si}$ & $\mathrm{Si}$ & $\mathrm{Si}$ & $\mathrm{Si}$ & $\mathrm{Si}$ & $\mathrm{Si}$ & $\mathrm{Si}$ & $\mathrm{Si}$ \\
PAS & $\mathrm{Si}$ & $\mathrm{Si}$ & $\mathrm{Si}$ & $\mathrm{Si}$ & $\mathrm{Si}$ & $\mathrm{Si}$ & $\mathrm{Si}$ & $\mathrm{Si}$
\end{tabular}

En la tabla 9 se muestran los resultados del proceso en su conjunto. Se puede observar que todos los integrantes del grupo reportaron que el proceso les ayudó para el reconocimiento emocional propio y de los demás tanto en lo referente al enojo como a la tristeza. Además aprendieron nuevas formas de expresar de manera constructiva sus emociones, tanto en el área verbal como conductual lo que coincide con lo que señala Read (1969) acerca de que, el dibujo promueve un estado emocionalmente sano.

\section{CONCLUSIONES}

Mediante el trabajo realizado a través del dibujo, se pudo constatar que promovió la expresión de los adolescentes y pudo ayudarles a satisfacer 
su necesidad de comunicación. Posibilitó la ampliación de su expresión de emociones y les ayudó en la promoción de un estado emocionalmente sano, pues los adolescentes fueron capaces de expresar lo que sintieron. Por otra parte, mediante los dibujos expresaron de manera gráfica lo más significativo en cada sesión. En ellos se destacan las expresiones faciales y verbales así como el trazo de figuras que expresa su experiencia. Los resultados obtenidos muestran que los adolescentes al irse familiarizando con el tema de las emociones en general y la forma que las experimentan en lo particular les permitió sensibilizarse hacia sí mismos y sus compañeros de grupo. Como esta fase se define fundamentalmente por una estructura de campo interpersonal, se dieron cuenta que al expresarse de manera violenta con sus compañeros generaban más conflictos. Como era de esperar, en un principio los adolescentes no eran sensibles ante las emociones de sus compañeros y el proceso psicoterapéutico les ayudó a reconocer que es importante tomar en cuenta las emociones propias y las de los demás para tener una mejor convivencia con las personas que les rodean. Tres meses después, en un seguimiento a corto plazo se pudo constatar que el proceso psicoterapéutico les ayudó a los adolescentes a la expresión no violenta de su enojo tanto en su núcleo familiar como escolar De manera que, los descubrimientos del estudio confirman que una vez que se pone en práctica la sensibilización y la reflexión focalizada en las experiencias emocionales de los adolescentes con la ayuda del dibujo como técnica facilitadora de su expresión verbal, en conjunto ayuda en el reconocimiento y la expresión constructiva de sus emociones y permite expresarse de manera no violenta en relaciones interpersonales sanas.

Este estudio presenta algunas limitaciones, principalmente la cantidad de los participantes. Sin embargo su importancia radica en que ofrece novedosas aportaciones referentes al trabajo de las emociones en 


\section{adolescentes en un contexto de psicoterapia grupal en el que mediante el uso del dibujo facilita la expresión emocional que como se pudo apreciar puede impactar de manera positiva las relaciones interpersonales en esta etapa del desarrollo. \\ REFERENCIAS}

I Almario, J. (2016). Una mirada existencial a la adolescencia. Colombia.

I Álvarez, A. (2009). Gestalt y violencia: cuando el encuentro se vuelve desencuentro. Uruguay.

I Albdour, M., Hong, J., Lewin, L. \& Yarandi, H. (2019). The Impact of Cyberbullying on Physical and Psychological Health of Arab American Adolescents. Journal of Immigrant and Minority Health.21, 706-715. doi.org/10.1007/s10903-018-00850-w

\Antúnez, Z. y Vinet, E. (2013). Problemas de salud mental en estudiantes de una universidad regional chilena. Revista Médica de Chile, 141(2), 209-216.

I Belsey, B. (2005). Cyberbullying: An emerging threat to the "always on" generation. http://www. cyberbullying.ca

$\checkmark$ Berelson, B. (1992). Content Analysis in Communications Research. New York.

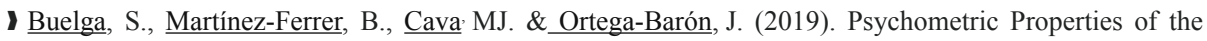
CYBVICS Cyber-Victimization Scale and Its Relationship with Psychosocial Variables. Social Scince. $8(1)$ doi.org/10.3390/socsci8010013

I Cain, D. \& Seeman, J. (2002). Humanistic Psychotherapies: Handbook of Research and Practice. Washington.

I Cannon, W. (1929). Bodily changes in pain, hunger, fear and rage, New York.

I Cervantes-Muñoz, MC. (2019). Efectos de la psicoterapia grupal humanista en el reconocimiento y la expresión emocional constructiva de adolescentes víctimas de ciberacoso. (Tesis de Maestría). Universidad Autónoma de Ciudad Juárez, Chihuahua, México.

\Chávez, G. (2017). Tecnología: Ciberbullying debe formar parte de la estrategia nacional de ciberseguridad. EXPANSIÓN. (2017/08/07) https://expansion.mx/tecnologia/2017/08/07/ciberbullying-debe-formar-partede-la-estrategia-nacional-de-ciberseguridad

\Cornejo, L. (2014). Manual de Terapia Gestáltica Aplicada a los Adolescentes. España.

I Cowie, H. (2013). El impacto emocional y las consecuencias del ciberacoso. Convives 3. http://www. fedadi.org/OTROS/Revista\%20CONVIVES\%20N_3\%20Abril\%202013.pdf\#page=16

I Damasio, A. (2005). Descartes error: emotion, reason, and the human brain. London.

I Damasio, A. (2010). Self comes to mind: constructing the conscious brain. New York.

I Darwin C. (1984). La expresión de las emociones en el hombre y los animales. Madrid.

I Delacroix, J. (2008). Encuentro con la psicoterapia: Una visión antropológica de la relación y el sentido de la enfermedad en la paradoja de la vida. Chile.

I Denzin, N. \& Lincoln, Y. (2018). The SAGE Handbook of Qualitative Research. USA.

$\checkmark$ Díaz, I. (2000). Bases de la terapia de grupo. México. 
I Díaz, C. y Navarro, P. (1998). Análisis de contenido: Métodos y técnicas cualitativas de investigación en ciencias sociales. Madrid.

I Ekman, P. (2003). Emotion Revealed: Recognizing faces and feelings to improve communication and emotional life. New York.

I Escobar-Echavarria, J., Montoya-González, L., Restrepo-Bernal, D. y Mejia-Rodríguez, D. (2017). Ciberacoso y comportamiento suicida. ¿Cuál es la conexión? A propósito de un caso. Revista Colombiana de Psiquiatría, 46. doi.org/10.1016/j.rcpeng.2017.09.004

I Finkelhor, D., Mitchell, K. y Wolak, J. (2000). Online Victimization: A Report on the Nation's Youth. National Center for Missing \& Exploited Children. http://www.unh.edu/ccrc/pdf/Victimization_Online Survey.pdf

I Greenberg, L. \& Paivio, S. (1997). Working with emotions in psychotherapy. New York.

I Garaigordobil, M. (2011). Prevalencia y consecuencias del cyberbullying: una revisión. International Journal of Psychology and Psychological Therapy. 11(2). http://www.federicofroebel.org/secondary/ presentacion-redes/prevalencia-yconsecuencias-del-cyberbullying.pdf

I Hsieh HF, Shannon SE. (2005) Three approaches to qualitative content analysis. Qual Health Res, 15(9):1277-1288.

1 Hauser, S. \& Safyer, A. (2010).Ego development and adolescent Emotions. Journal of Research on Adolescence, 4(4). 487-502. DOI: 10.1207/s15327795jra0404_3

I Hernández, A. (2006). Las hebras para hilvanar la vida: el dibujo del dolor. Arteterapia, Papeles de arteterapia y educación artística para la inclusión social, 1, 79-96. https://doi.org/-

I Hernández, R., Fernández, C. y Baptista, P. (2016). Metodología de la Investigación. México.

$\checkmark$ Hurlock E. (1980). Psicología de la adolescencia. México. Paidós Psicología Evolutiva.

I Ibarra, E. (2014). Protección de niños en la red: Sexting, Ciberbullying y Pornografia Infantil. México.

I James, W. (1994). Principios de psicología. México.

I Jackson, S. \& Goosssen, L. (2016). Handbook of adolescent Development. New York.

I Keltner, D., Moffit, T., \& Stouthamer-Loeber, M. (1995). Facial expression of emotion and psychopathology in adolescent boys. Journal and Abnormal Psychology, 104(4), 644-652.

I Kepner, J. (1992). Proceso corporal. Un enfoque Gestalt para el trabajo corporal en psicoterapia. México.

I Kerlinger. F. y Lee, H. (2008). Investigación del comportamiento humano: métodos de investigación en ciencias sociales. México.

I Kowalski, R., \& Limber, S. P. (2013). Psychological, physical, and academic correlates of cyberbullying and traditional bullying. Journal of Adolescent Health, Journal of Adolescent Health, 53(1), 13-20.

I Kriz, J. (2001). Corrientes fundamentales en psicoterapia. Argentina.

I Lane, R., Ryan, L., \& Greenberg, L. (2015). Memory reconsolidation, emotional arousal, and the process of change in psychotherapy: New insights from brain science. Behavior and Brian Science, 38. Doi: 101017/S0140525X14000041.Epub2014May15.

I Latner, J. (2007). Fundamentos de la Gestalt. Chile.

I Linn, S. y Garske, J. (1988). Psicoterapias contemporáneas: Modelos y métodos. España. 
I McLeod, J. (2010). Case Study Research in Counselling and Psychotherapy. California.

I Mapua, J. (2018). Coping with cyberbullyng. New York.

I Marsh, H. (1989). Age and sex effects in multiple dimensions of self-concept: Preadolescence to early adulthood. Journal of Educational Psychology, 81(3), 417-430. http://dx.doi.org/10.1037/0022$\underline{0663.81 .3 .417}$

May, N. y Pope, C. (1995). Rigor and Qualitative research. Clinical Research, 109-112.

I Mayring (2000). Quanlitative Content Analysis. Forum: Qualitative Social Research Sozialforschung, 1 (20), http://www.qualitative-research.net/index.php/fqs/article/view/1089/2385\#g4

I McConville, Mark. (1995). Adolescence: Psychotherapy and the emergent self. San Francisco.

I Oaklander, V. (2010). Ventana A Nuestros Niños Terapia Gestáltica Para Niños Y Adolescentes. (6a. Ed.), Chile.

I Oaklander, V. (2006). El Tesoro Escondido. Chile.

I Olweus, D. (1997). Bully/victim problem in school: Facts and intervention. European Journal of Psychology of Education 12(4). https://pdfs.semanticscholar.org/13e7/755d6e9ec2c6c00d7b7f86f685b7652ce952.pdf

$\checkmark$ Olweus, D. (1994). Bullying at School: Basic Facts and Effects of a School Based Intervention Program. Journal of Child Psychology and Psychiatry, 35 (7). doi.org/10.1111/j.1469-7610.1994.tb01229.x

I Ortega, R., Calmaestra, J. y Mora-Merchán, J. (2007). Cuestionario de Ciberbullying. Manuscrito inédito.

I Ortega, R., Elipe, P., Mora-Merchan, J.A., Genta, M.L., Brighi, A., Guarini, A. \& Tippett, N. (2012). The emotional impact of bullying and cyberbullying on victims: A Europeancross-national study. Aggressive Behavior, 38, 342-356.

I Organización Mundial de la Salud (2018). Desarrollo en la adolescencia. http://www.who.int/maternal child_adolescent/topics/adolescence/dev/es/

I Perls, F. (2011). El Enfoque Gestáltico \& Testimonios de Terapia. Chile.

I Perls, F., Hefferline, R. y Goodman, P. (2006). Terapia Gestalt: la excitación y el crecimiento de la personalidad humana. Madrid: Sociedad de Cultura Valle-Inclán.

I Palmero, F. (2000). Emoción. Breve reseña del papel de la cognición y el estado afectivo. Revista Española de Motivación y Emoción. 1. http://reme.uji.es/articulos/apalmf245161299/texto.html

I Plutchik, R. (1991). The Emotions. Nueva York.

I Polster, E. y Polster, M. (2008). Terapia gestáltica: Perfiles de teoría y práctica. Buenos Aires.

$\checkmark$ Read, H. (1969). Educación por el arte. Barcelona: Paidós.

I Rieffe, C., Villanueva, L., Adrián, y J., Górriz, A. (2009). Quejas somáticas, estados de ánimo y consciencia emocional en adolescentes. Psicothema, 21. http://www.redalyc.org/articulo.oa?id=72711821019ISSN 0214-9915

$\checkmark$ Salmerón, M. e Inostroza, C. (2017). Repercusiones sobre la salud del maltrato entre iguales coso escolar y ciberacoso. Revista de Estudios de Juventud, 115. https://dialnet.unirioja.es/servlet/ articulo? codigo $=6215271$

I Soutullo, C. y Mardomingo, M. J. (2009). Manual de psiquiatría del niño y el adolescente. España.

1 Sroufe, L. A. (2000). Desarrollo Emocional. México. 
I Stern, A. (1977). La expresión. Barcelona.

I Strauss, A. y Colbin, J. (2002). Bases de la investigación cualitativa: técnicas y procedimientos para desarrollar la Teoría Fundamentada. Antioquía.

I Sociedad Mexicana de Psicología SMP (2009). Código ético del psicólogo. México.

- Taylor SJ, Bogdan R. (1990) Introducción a los métodos cualitativos de investigación. Barcelona.

I Weiner, I. V. (1992). Métodos en psicología clínica. México.

I Yalom, I. (1986). Teoría y práctica de la psicoterapia de grupo. México.

I Yontef, G. (2009). Proceso y Diálogo en Psicoterapia Gestáltica, Chile.

Envío a dictamen: 29 de junio de 2020

Reenvío: 5 de agosto de 2020 Aprobación:24 de agosto de 2020

María del Consuelo Cervantes-Muñoz. Maestra en Psicología, Becada por el Consejo Nacional de Ciencia y Tecnología. Integrante del Núcleo Académico Básico de la Maestría en Psicoterapia Humanista y Educación para la Paz de la Universidad Autónoma de Ciudad Juárez. E-mail: ccervantes67@hotmail.com

María Nieves González Valles. Profesora/Investigadora de Tiempo completo en el Instituto de Ciencias Sociales y Administración de la Universidad Autónoma de Ciudad Juárez. E-mail: mgonzale@uacj.mx

Iris Rubí Monroy Velasco. Profesora/Investigadora de Tiempo Completo de la Facultad de Psicología de la Universidad Autónoma de Coahuila. E-mail: iris.monroy@uadec.edu.mx 